\title{
n-3 fatty acid-enriched parenteral nutrition regimens in elective surgical and ICU patients: a meta-analysis
}

\author{
Lorenzo Pradelli i*, Konstantin Mayer ${ }^{2}$, Maurizio Muscaritoli ${ }^{3}$ and Axel R Heller ${ }^{4}$
}

\begin{abstract}
Introduction: Previous studies and a meta-analysis in surgical patients indicate that supplementing parenteral nutrition regimens with n-3 polyunsaturated fatty acids (PUFAs), in particular eicosapentaenoic acid (EPA) and docosahexaenoic acid (DHA), is associated with improved laboratory and clinical outcomes in the setting of hyperinflammatory conditions. Refined or synthetic fish oils are commonly used as a source of EPA and DHA. The objective of the present meta-analysis was to evaluate n-3 PUFA-enriched parenteral nutrition regimens in elective surgical and intensive care unit (ICU) patients.

Methods: Medline was searched for randomized controlled trials comparing n-3 PUFA-enriched lipid emulsions with standard non-enriched lipid emulsions (i.e. soybean oil, MCT/LCT or olive/soybean oil emulsions) in surgical and ICU patients receiving parenteral nutrition. Extracted data were pooled by means of both random and fixed effects models, and subgroup analyses were carried forward to compare findings in ICU versus non-ICU patients.

Results: A total of 23 studies ( $n=1502$ patients: $n=762$ admitted to the ICU) were included. No statistically significant difference in mortality rate was found between patients receiving $n-3$ PUFA-enriched lipid emulsions and those receiving standard lipid emulsions $(R R=0.89 ; 0.59,1.33)$, possibly reflecting a relatively low underlying mortality risk. However, n-3 PUFA-enriched emulsions are associated with a statistically and clinically significant reduction in the infection rate $(R R=0.61 ; 0.45,0.84)$ and the lengths of stay, both in the ICU $(-1.92 ;-3.27,-0.58)$ and in hospital overall $(-3.29 ;-5.13,-1.45)$. Other beneficial effects included reduced markers of inflammation, improved lung gas exchange, liver function, antioxidant status and fatty acid composition of plasma phospholipids, and a trend towards less impairment of kidney function.
\end{abstract}

Conclusions: These results confirm and extend previous findings, indicating that n-3 PUFAs-enriched parenteral nutrition regimens are safe and effective in reducing the infection rate and hospital/ICU stay in surgical and ICU patients.

\section{Introduction}

The role of polyunsaturated fatty acids (PUFA) in the modulation of biologic activities was identified some decades ago, starting from the first studies on the lower cardiovascular risk found in populations with an extremely high intake of n-3 PUFA such as eicosapentaenoic acid (EPA), and docosahexaenoic acid (DHA) [1,2]. In addition to being structural constituents of cell membranes, PUFA are precursors of biological mediators involved in the

\footnotetext{
* Correspondence: I.pradelli@adreshe.com

${ }^{1}$ AdRes HE\&OR, Piazza Carlo Emanuele II 19, I-10123, Turin, Italy

Full list of author information is available at the end of the article
}

regulation of many physiological functions, including immune response, blood pressure regulation, cell proliferation, blood clotting, and inflammation $[3,4]$. The balance between $n-3$ and n- 6 PUFA is important, as mediators derived from the n-6 PUFA (mainly arachidonic acid, AA) favour an inflammatory response, while mediators stemming from n-3 PUFA such as EPA and DHA exert less pro-inflammatory actions.

Intravenous lipid emulsions have been established for many years as an integral part of parenteral nutrition due to their high energy density and low osmolarity. These emulsions are traditionally based on vegetable oils that are rich in n-6 fatty acids, such as soybean oil. In recent
() Biomed Central

C 2012 Pradelli et al.; licensee BioMed Central Ltd. This is an open access article distributed under the terms of the Creative Commons Attribution License (http://creativecommons.org/licenses/by/2.0), which permits unrestricted use, distribution, and reproduction in any medium, provided the original work is properly cited. 
years, many trials have explored whether parenteral nutrition regimens supplemented with n-3 PUFA may be beneficial in those clinical conditions that are characterized by an inflammatory over-response, for example, sepsis or pancreatitis, and after major abdominal surgery. Refined or synthetic fish oils are commonly used as a source of EPA and DHA and have been incorporated into a new generation of mixed lipid emulsions.

Chen et al. published a meta-analysis of trials conducted in patients undergoing major abdominal surgery and found evidence that parenteral n-3-enriched lipid emulsions in the setting of total parenteral nutrition are beneficial in terms of relevant clinical outcomes, such as infection rate and hospital length of stay (LOS) [5]. However, several studies evaluating the effects of fish oilbased emulsions in clinical conditions in which such hyper-inflammation is a characteristic were omitted from this meta-analysis. Moreover, additional clinical studies have been published in the interim.

The objective of our study was to provide an updated and more extensive analysis of the available evidence on the clinical efficacy and safety of n-3 PUFA-enriched parenteral lipid emulsions in elective surgical and ICU patients, as compared to standard (non-enriched) lipid emulsions, namely, soybean oil, medium-chain triglycerides (MCT)/long-chain triglycerides (LCT) or olive/ soybean oil emulsions.

\section{Materials and methods}

The Pubmed database was searched for relevant papers with the following search string: ("Fatty Acids, Omega-3"[Mesh] OR "Fish oil") AND "Parenteral Nutrition"[Mesh] AND ("Surgical Procedures, Operative"[Mesh] OR "Sepsis"[Mesh] OR "Systemic Inflammatory Response Syndrome"[Mesh] OR "Intensive Care Units"[Mesh]). Identified papers were checked for coherence with the defined inclusion criteria, and the reference list of those deemed relevant was manually searched for further relevant studies.

To be included in the present analysis, the retrieved papers were required to report on the results of randomized clinical trials (RCTs) comparing n-3 PUFAenriched lipid emulsions with standard non-enriched lipid emulsions (that is, soybean oil, MCT/LCT or olive/ soybean oil emulsions) in adult ICU patients and/or in elective surgery patients, in terms of clinical outcomes, markers of inflammation and antioxidant status, fatty acid composition of plasma phospholipids, and/or routine laboratory parameters (Table 1).

Identified papers were checked to identify whether results of a single study were published more than once, in order to avoid double imputation. In such instances, the secondary publications were considered only for parameters that were not reported by the main publication.
Results were analysed both overall, and by subgroup (nonICU patients versus ICU patients). Allocation to the ICU subgroup was driven by the explicit mentioning in the published paper of an ICU stay, either in the Methods or in the Results section.

Data were extracted from the text, tables and figures of the original published papers, without any effort to retrieve further data by contacting the authors. Mean and SD were used for the meta-analysis: where these were not reported, they were calculated by fitting an appropriate distribution to the available data. In case of a missing SD only, this value was imputed based on the average $\mathrm{SD} /$ mean ratio of included studies reporting on the same parameter. In the case of more than half of the studies not reporting the SD, analysis was not conducted on that parameter. Data pooling was performed with the use of classical meta-analytic methodology, using the RevMan 5.1 software developed for the Cochrane Collaboration. The primary analysis was conducted with random effects models, with Mantel-Haenszel weighting for binary outcomes, and inverse variance weighting for continuous parameters. Exploratory analyses based on fixed effects models were also conducted.

\section{Results}

The original search (August 2011) in PubMed yielded 52 hits. Of these, thirty-seven publications were excluded, as they were not consistent with the inclusion criteria: thirteen were not clinical trials (eleven reviews, one case report, one drug development study), three did not report on any of the analysed outcomes, eight were not randomized, in two the supplementation was administered enterally or orally, in four the study population was not consistent with the specified criteria, and finally, seven studies were not conducted in human subjects. The manual search of the reference lists of the remaining fifteen studies yielded a further eight relevant studies (Additional file 1).

Thus, a total of 23 studies, including a total of 1,502 patients, were included in the meta-analysis: 13 [6-18] of these were conducted in patients admitted to the ICU $(\mathrm{n}=762)$, and 10 [19-28] in patients undergoing major abdominal surgery and not admitted to ICU $(n=740)$ (Table 2).

Checking for duplicate publication of the same data revealed that the studies by Antebi et al. [6] and Grimm et al. [20] were subgroup analyses of the same study fully published by Mertes et al. [27]. We therefore excluded from the analyses the values reported Antebi et al. [6] for aspartate aminotransferase (AST), alanine aminotransferase (ALT) and triglyceride levels, and the data on hospital LOS recorded in Grimm et al. [20], as all of these parameters were already in the Mertes et al. publication [27]. Hospital LOS data were reported as 


\begin{tabular}{|c|c|}
\hline Outcome & Definition \\
\hline Mortality & Number of deaths as reported/patients receiving at least one treatment \\
\hline Infection rate & Number of nosocomial infections/patients receiving at least one treatment dose \\
\hline Hospital length of stay $(L O S)^{a}$ & Mean (SD) number of hospital days from hospitalization (or intervention) to discharge \\
\hline ICU LOS & Mean (SD) number of ICU days \\
\hline Transfused blood units & Standard units \\
\hline Oxygenation index & Mean (SD) ratio of partial oxygen pressure $\left(\mathrm{PO}_{2}\right)$ : inspired oxygen fraction $\left(\mathrm{FiO}_{2}\right)$ \\
\hline \multicolumn{2}{|l|}{ Serum parameters } \\
\hline Alpha-tocopherol & Mean (SD) serum concentration, $\mu \mathrm{mol} / \mathrm{L}$ \\
\hline Aspartate aminotransferase (AST) & Mean (SD) serum concentration, IU/L \\
\hline Alanine aminotransferase (ALT) & Mean (SD) serum concentration, IU/L \\
\hline Bilirubin & Mean (SD) serum concentration, mg/dL \\
\hline C-reactive protein (CRP) & Mean (SD) serum concentration, $\mathrm{mg} / \mathrm{dL}$ \\
\hline Creatinine & Mean (SD) serum concentration, $\mathrm{mg} / \mathrm{dL}$ \\
\hline Interleukin (IL)-6 change & Mean (SD) difference in serum IL-6 levels between end and beginning of infusion, $\mathrm{pg} / \mathrm{mL}$ \\
\hline Lactate & Mean (SD) serum concentration, $\mathrm{mmol} / \mathrm{L}$ \\
\hline Triglycerides & Mean (SD) serum concentration, $\mathrm{mg} / \mathrm{dL}$ \\
\hline Urea & Mean serum concentration, $\mathrm{mmol} / \mathrm{L}$ \\
\hline \multicolumn{2}{|l|}{ Other laboratory parameters } \\
\hline Leukotriene B5 (LTB5) & Ex-vivo production by leukocytes ${ }^{b}$, \\
\hline \multicolumn{2}{|l|}{ Leukotriene B4 (LTB4) } \\
\hline LTB5/LTB4 ratio & Ex-vivo production by leukocytes \\
\hline Eicosapentaenoic acid (EPA) & Content in plasma phospholipids, (\% of total concentration) \\
\hline \multicolumn{2}{|l|}{ Docosahexaenoic acid (DHA) } \\
\hline \multicolumn{2}{|l|}{ Arachidonic acid (AA) } \\
\hline Prothrombin time, PT (Quick) & Laboratory standard \\
\hline Partial thromboplastin time (PTT) & Laboratory standard \\
\hline Platelets & Count, $\times 10^{3} / \mu \mathrm{L}$ \\
\hline
\end{tabular}

${ }^{\mathrm{a}}$ When reported as median and interquartile range, data were transformed into mean (SD) by fitting a Weibull distribution. ${ }^{\mathrm{b}}$ Analysed in terms of standardised difference as expressed in heterogeneous measurement units.

median and interquartile range by more than one study: the corresponding mean and SD values were estimated after fitting a Weibull distribution to reported data.

\section{Clinical Outcomes}

The results of the pooled analyses with the random effects models (Table 3 ) indicate no statistically significant difference in mortality rate between patients receiving $n-3$ PUFA-enriched lipid emulsions and those receiving the standard lipid emulsions, soybean oil, MCT/LCT or olive/ soybean oil emulsions. The time point at which mortality was assessed varied: while $28 / 30$ days mortality was the most commonly used definition $[7,9,16,18]$, in-hospital mortality was analysed in one report [19], and in the remaining studies $[14,17,26,27]$ the endpoint for this outcome was not specified. This lack of clarity/homogeneity may somewhat hamper the interpretation of presented results.

However, n-3 PUFA-enriched regimens are associated with a statistically and clinically significant reduction in the infection rate (Figure 1) and LOS, both in the ICU
(Figure 2) and in hospital overall (Figure 3). If only ICU patients' data are considered, the reduction in infection rate is not statistically significant.

Lung gas exchange (oxygenation index), measured in only two studies in septic ICU patients $[7,16]$, was significantly increased in patients receiving 3 PUFA-enriched parenteral nutrition regimens. No statistically significant differences between treatments could be detected for bleeding-related outcomes, including blood transfusion requirements.

\section{Markers of inflammation, antioxidant status and fatty acid composition of plasma phospholipids}

Use of n-3 PUFA-enriched emulsions significantly increases the serum concentration of alpha-tocopherol and the percentage content of EPA (Figure 4) and DHA (Figure 5) in phospholipids. However, the content of AA in phospholipids was unchanged by $n-3$ PUFA enrichment.

There was a significantly greater reduction in IL-6 and a shift in the generation of leukotrienes towards the leukotriene- 5 series, as indicated by the significant absolute 
Table 2 Studies evaluating n-3 PUFA-enriched lipid emulsions for parenteral nutrition and reported outcomes/ measured parameters

\begin{tabular}{|c|c|c|c|c|c|}
\hline Study & Setting (n) & $\begin{array}{l}\text { n-3 PUFA- } \\
\text { enriched lipid } \\
\text { emulsion }\end{array}$ & $\begin{array}{l}\text { Standard } \\
\text { lipid } \\
\text { emulsion }\end{array}$ & Clinical outcomes & Laboratory outcomes \\
\hline \multicolumn{6}{|l|}{$\overline{\text { ICU patients }}{ }^{\mathrm{a}}(\mathrm{n}=762)$} \\
\hline Antebi $2004[6]$ & Major surgery (20) & $\mathrm{SO} / \mathrm{MCT} / \mathrm{OO} / \mathrm{FO}^{\mathrm{b}}$ & so & & $\mathrm{AST}^{c}, \mathrm{ALT}^{c}, \mathrm{CRP}$, alpha-T,TG \\
\hline Barbosa $2010[7]$ & Sepsis (23) & SO/MCT/n-3 TGs ${ }^{d}$ & $\mathrm{sO} / \mathrm{OO}$ & $\begin{array}{l}\text { H LOS, ICU LOS, } \\
\text { Mortality }\end{array}$ & $\begin{array}{l}\text { CRP, EPA, DHA, AA, LTB4, AST, } \\
\text { ALT, Bilirubin, OI, IL-6, PTT, Lac }\end{array}$ \\
\hline Berger 2008 [8] & $\begin{array}{l}\text { Abdominal aortic } \\
\text { aneurysm (24) }\end{array}$ & $\mathrm{SO} / \mathrm{MCT} / \mathrm{n}-3 \mathrm{TGS}^{\mathrm{d}}$ & $\mathrm{sO} / \mathrm{OO}$ & $\begin{array}{l}\text { Mortality, H LOS, ICU } \\
\text { LOS }\end{array}$ & EPA, DHA, AA, alpha-T, CRP, TG \\
\hline Friesecke 2008 [9] & Critical medical (165) & $\mathrm{SO}+\mathrm{FO}^{\mathrm{e}}$ & $\mathrm{sO} / \mathrm{OO}$ & $\begin{array}{l}\text { Mortality, Infection rate, } \\
\text { ICU LOS, Bleeding events }\end{array}$ & $I L-6^{f} T B U$ \\
\hline Heller $2004[10]$ & Elective colorectal (44) & $\mathrm{SO}+\mathrm{FO}^{\mathrm{e}}$ & so & ICU LOS & $\begin{array}{l}\text { AST, ALT, CRP, Bilirubin, PT } \\
\text { (Quick), PTT, TBU }\end{array}$ \\
\hline Morlion 1996 [11] & Gastric carcinoma (20) & $\mathrm{SO}+\mathrm{FO}^{\mathrm{e}}$ & so & & AA, EPA, DHA, LTB5, LTB4 \\
\hline $\begin{array}{l}\text { Piper } 2009 \\
{[12]}\end{array}$ & $\begin{array}{l}\text { Major abdominal or } \\
\text { craniomaxillofacial } \\
\text { surgery (44) }\end{array}$ & $\mathrm{SO} / \mathrm{MCT} / \mathrm{OO} / \mathrm{FO}^{\mathrm{b}}$ & $\mathrm{sO} / \mathrm{OO}$ & & AST, ALT, TG \\
\hline Roulet 1997 [13] & $\begin{array}{l}\text { Elective oesophagectomy } \\
\text { (19) }\end{array}$ & $\mathrm{SO}+\mathrm{FO}^{\mathrm{e}}$ & so & & EPA, DHA, AA, BT \\
\hline Sabater 2011 [14] & $\begin{array}{l}\text { Acute respiratory distress } \\
\text { syndrome (44) }\end{array}$ & SO/MCT/n-3 TGs ${ }^{d}$ & so & Mortality & LTB4 \\
\hline Wachtler 1997 [15] & $\begin{array}{l}\text { Elective abdominal } \\
\text { surgery (40) }\end{array}$ & SO/MCT/n-3 TGs ${ }^{d}$ & $\mathrm{sO} / \mathrm{OO}$ & $\begin{array}{l}\text { Infection rate, H LOS, } \\
\text { ICU LOS }\end{array}$ & LTB4, LTB5, LTB ratio, IL-6 ${ }^{f}$ \\
\hline Wang 2008 [16] & $\begin{array}{l}\text { Severe acute pancreatitis } \\
(40)\end{array}$ & $\mathrm{SO}+\mathrm{FO}^{\mathrm{e}}$ & so & $\begin{array}{l}\text { Mortality, Infection rate, } \\
\text { H LOS, ICU LOS }\end{array}$ & EPA, CRP, OI \\
\hline Weiss 2002 [17] & $\begin{array}{l}\text { Gastrointestinal surgery } \\
\text { (23) }\end{array}$ & $\mathrm{SO}+\mathrm{FO}^{\mathrm{e}}$ & so & $\begin{array}{l}\text { Mortality, Infection rate, } \\
\text { H LOS, ICU LOS }\end{array}$ & $\mathrm{IL}-6^{\mathrm{g}}$ \\
\hline Wichmann 2007 [18] & $\begin{array}{l}\text { Major intestinal surgery } \\
(256)\end{array}$ & SO/MCT/n-3 TGs ${ }^{d}$ & so & $\begin{array}{l}\text { Mortality, Infection rate, } \\
\text { H LOS, ICU LOS }\end{array}$ & $\begin{array}{l}\text { AST, Bilirubin, TG, CRP, LTB5, LTB } \\
\text { ratio, alpha-T, EPA, PT (Quick), Cr }\end{array}$ \\
\hline \multicolumn{6}{|c|}{ Elective surgery, non-ICU patients $(n=740)$} \\
\hline Badia-Tahull 2010 [19] & $\begin{array}{l}\text { Major gastrointestinal } \\
\text { surgery (27) }\end{array}$ & $\mathrm{SO}+\mathrm{FO}^{\mathrm{e}}$ & sO/OO) & $\begin{array}{l}\text { Mortality, } \mathrm{H} \text { LOS, } \\
\text { Infection rate }\end{array}$ & $\mathrm{ALT}, \mathrm{CRP}, \mathrm{TBU}, \mathrm{Cr}, \mathrm{PIU}$ \\
\hline Grimm 2006 [20] & $\begin{array}{l}\text { Radical colorectal cancer } \\
\text { resection (33) }\end{array}$ & $\mathrm{SO} / \mathrm{MCT} / \mathrm{OO} / \mathrm{FO}^{\mathrm{b}}$ & so & $\mathrm{H} \operatorname{LOS}^{\mathrm{C}}$ & $\begin{array}{l}\text { alpha-T, AA, EPA, DHA, LTB4, } \\
\text { LTB5, LTB ratio }\end{array}$ \\
\hline Jiang 2010 [21] & $\begin{array}{l}\text { Gastrointestinal } \\
\text { malignancy (203) }\end{array}$ & $\mathrm{SO}+\mathrm{FO}^{\mathrm{e}}$ & so & $\begin{array}{l}\text { H LOS, Infection rate, } \\
\text { Bleeding events }\end{array}$ & $\mathrm{IL}-6, \mathrm{CrCl}$ \\
\hline Klek 2005[22] & $\begin{array}{l}\text { Major abdominal surgery } \\
\text { (58) }\end{array}$ & $\mathrm{SO}+\mathrm{FO}^{\mathrm{e}}$ & $\mathrm{sO} / \mathrm{OO}$ & H LOS, Infection rate & $\mathrm{ALT}, \mathrm{AST}, \mathrm{Cr}, \mathrm{PIU}$ \\
\hline Koeller 2003 [23] & $\begin{array}{l}\text { Major abdominal surgery } \\
(30)\end{array}$ & SO/MCT/n-3 TGs ${ }^{d}$ & so & & LTB4, LTB5, LTB ratio \\
\hline Liang 2008 [24] & Colorectal cancer (41) & $\mathrm{SO}+\mathrm{FO}^{\mathrm{e}}$ & so & $\begin{array}{l}\text { Mortality, Infection rate, } \\
\text { H LOS }\end{array}$ & $\mathrm{IL}-6$ \\
\hline Linseisen 2000 [25] & $\begin{array}{l}\text { Major abdominal surgery } \\
\text { (33) }\end{array}$ & $\mathrm{SO} / \mathrm{MCT} / \mathrm{n}-3 \mathrm{TGs}^{\mathrm{cd}}$ & so & & alpha-T, AA, EPA, DHA \\
\hline Makay 2011 [26] & Major gastric surgery (26) & $\mathrm{SO}+\mathrm{FO}^{\mathrm{e}}$ & so & $\begin{array}{l}\text { Mortality, Infection rate, } \\
\text { H LOS }\end{array}$ & AST, ALT, Cr, PIU; LaC \\
\hline Mertes 2006 [27] & Abdominal surgery (249) & $\mathrm{SO} / \mathrm{MCT} / \mathrm{OO} / \mathrm{FO}^{\mathrm{b}}$ & so & Mortality, H LOS & AST, ALT, TG, Bilirubin \\
\hline Senkal 2007 [28] & $\begin{array}{l}\text { Major abdominal cancer } \\
(40)\end{array}$ & SO/MCT/n-3 TGs ${ }^{d}$ & $\mathrm{sO} / \mathrm{OO}$ & Infection rate & $\mathrm{AA}, \mathrm{EPA}, \mathrm{DHA}$ \\
\hline
\end{tabular}

${ }^{a}$ Allocation to the ICU subgroup was driven by explicit mention in the published paper of an ICU stay, in the Methods or Results sections of the original article. ${ }^{b}$ SMOFlipid 20\% (Fresenius Kabi): $1000 \mathrm{ml}$ of emulsion contains: soya-bean oil, refined $60.0 \mathrm{~g}$, triglycerides, medium-chain, $60.0 \mathrm{~g}$, olive oil, refined $50.0 \mathrm{~g}$, FO, rich in n-3-acids $30.0 \mathrm{~g}$. 'Not used for meta-analysis as data are from a subgroup of Mertes et al.[27]. 'Lipoplus 20\% (B.Braun): 1000 ml of emulsion contains: mediumchain triglycerides: $100.0 \mathrm{~g}$, soybean oil, refined: $80.0 \mathrm{~g}$, N-3-acid triglycerides: $20.0 \mathrm{~g}$. ${ }^{\mathrm{e}}$ Omegaven $10 \%$ fish oil emulsion (Fresenius Kabi): $100 \mathrm{ml}$ emulsion contains highly refined fish oil $10.0 \mathrm{~g}$ containing eicosapentaenoic acid (EPA) 1.25 to $2.82 \mathrm{~g}$, docosahexaenoic acid (DHA) 1.44 to $3.09 \mathrm{~g}$, dl- $\alpha$-tocopherol (as antioxidant) 0.015 to $0.0296 \mathrm{~g}$. ${ }^{\mathrm{f}}$ Data reported by graph only or qualitatively. ${ }^{9}$ No SD reported. AA, (\%) content of arachidonic acid in serum/cellular membranes; alpha-T, alpha-tocopherol; ALT, alanine aminotransferase; AST, aspartate aminotransferase; BT, bleeding time; $\mathrm{Cr}$, serum creatinine; CrCl, creatinine clearance; CRP, C-reactive protein; $\mathrm{DHA}$,(\%) docosahexaenoic acid content in serum/cellular membranes; EPA, (\%) eicosapentaenoic acid content in serum/cellular membranes; FO, fish oil emulsion; Lac, lactate; (H) LOS, (Hospital) length of stay; ICU, intensive care unit; LTB, leukotriene B; LTB ratio, LTB5: LTB4; ( $n-3$ ) TGs, ( $\mathrm{n}-3$ ) triglycerides; MCT, medium chain triglycerides; Ol, oxygenation index; OO, olive oil emulsion; PIU, serum urea; PT, prothrombin time; PTT, partial thromboplastin time; SO, soybean oil emulsion; TBU, transfused blood units. 
Table 3 n-3 PUFA-enriched versus standard parenteral lipid emulsions for parenteral nutrition: random effects metaanalysis

\begin{tabular}{|c|c|c|c|}
\hline Outcome & Studies & Patients (n) & Effect estimate \\
\hline Mortality, overall RR & 10 & 847 & $0.89(0.59,1.33)$ \\
\hline ICU patients & 7 & 547 & $0.94(0.61,1.45)$ \\
\hline Non-ICU patients & 3 & 300 & $0.58(0.18,1.84)$ \\
\hline Infection rate, overall RR & 11 & 919 & $0.61(0.45,0.84)^{*}$ \\
\hline ICU patients & 5 & 524 & $0.71(0.45,1.12)$ \\
\hline Non-ICU patients & 6 & 395 & $0.53(0.34,0.82)^{*}$ \\
\hline Hospital LOS, overall MD & 15 & 1169 & $-3.29(-5.13,-1.45)^{*}$ \\
\hline ICU patients & 8 & 615 & $-5.17(-8.35,-1.99)^{*}$ \\
\hline Non-ICU patients & 7 & 554 & $-1.86(-3.13,-0.59)^{*}$ \\
\hline ICU LOS, MD & 8 & 615 & $-1.92(-3.27,-0.58)^{*}$ \\
\hline CRP, overall MD & 7 & 432 & $-11.28(-24.71,2.16)$ \\
\hline ICU patients & 6 & 405 & $-9.76(-23.57,4.04)$ \\
\hline Non-ICU patients & 1 & 27 & $-46.00(-108.12,16.12)$ \\
\hline IL-6 change, MD & 3 & 105 & $37.70(20.23,55.16)^{*}$ \\
\hline Oxygenation index, MD & 2 & 61 & $50.04(10.99,89.09)^{*}$ \\
\hline Serum lactate, MD & 2 & 47 & $-0.29(-1.38,0.80)$ \\
\hline LTB5, overall SMD & 5 & 183 & $2.86(1.22,4.50)^{*}$ \\
\hline ICU patients & 3 & 120 & $3.35(0.54,6.16)^{*}$ \\
\hline Non-ICU patients & 2 & 63 & $2.14(0.42,3.85)^{*}$ \\
\hline LTB4, overall SMD & 6 & 188 & $-0.47(-1.18,0.23)$ \\
\hline ICU patients & 4 & 125 & $-0.85(-1.42,-0.27)^{*}$ \\
\hline Non-ICU patients & 2 & 63 & $0.34(-1.25,1.92)$ \\
\hline LTB ratio, overall MD & 4 & 163 & $0.07(0.05,0.09)^{*}$ \\
\hline ICU patients & 2 & 100 & $0.11(0.01,0.22)^{*}$ \\
\hline Non-ICU patients & 2 & 63 & $0.06(0.05,0.07)^{*}$ \\
\hline EPA, overall SMD & 9 & 271 & $4.12(2.99,5.25)^{*}$ \\
\hline ICU patients & 6 & 165 & $4.65(2.70,6.60)^{*}$ \\
\hline Non-ICU patients & 3 & 106 & $3.64(2.65,4.64)^{*}$ \\
\hline DHA, overall SMD & 7 & 171 & $1.84(0.65,3.03)^{*}$ \\
\hline ICU patients & 4 & 65 & $2.82(0.17,5.46)^{*}$ \\
\hline Non-ICU patients & 3 & 106 & $1.33(-0.11,2.78)$ \\
\hline Arachidonic acid, overall SMD & 6 & 171 & $0.22(-0.20,0.64)$ \\
\hline ICU patients & 3 & 65 & $0.35(-0.14,0.84)$ \\
\hline Non-ICU patients & 3 & 106 & $0.14(-0.62,0.900$ \\
\hline Alpha-tocopherol, overall MD & 5 & 170 & $12.33(8.73,15.93)^{*}$ \\
\hline ICU patients & 3 & 104 & $10.08(5.39,14.76)^{*}$ \\
\hline Non-ICU patients & 2 & 66 & $15.25(14.15,16.35)^{*}$ \\
\hline Prothromin time (Quick), MD & 2 & 300 & $0.43(-2.62,3.47)$ \\
\hline Partial thromboplastin time, MD & 2 & 65 & $10.71(-30.08,51.51)$ \\
\hline Transfused blood units, SMD & 2 & 209 & $-0.05(-0.32,0.22)$ \\
\hline Platelet count & 4 & 160 & $-6.32(-31.40,18.77)$ \\
\hline Triglyceride level, overall MD & 5 & 567 & $10.40(-13.53,34.34)$ \\
\hline ICU patients & 4 & 368 & $14.16(-13.12,41.44)$ \\
\hline Non-ICU patients & 1 & 199 & $-0.89(-23.26,21.48)$ \\
\hline Serum creatinine, SMD & 3 & 309 & $-3.01(-7.11,1.08)$ \\
\hline Serum urea, SMD & 2 & 53 & $-0.11(-0.30,0.08)$ \\
\hline AST, overall MD & 7 & 656 & $-10.05(-18.81,-1.29)^{*}$ \\
\hline ICU patients & 4 & 373 & $-10.11(-27.31,7.10)$ \\
\hline Non-ICU patients & 3 & 283 & $-8.37(-17.36,0.61)^{*}$ \\
\hline ALT, overall MD & 7 & 482 & $-9.85(-17.49,-2.21)^{*}$ \\
\hline ICU patients & 3 & 109 & $-18.18(-21.68,-14.68)^{*}$ \\
\hline
\end{tabular}


Table 3 n-3 PUFA-enriched versus standard parenteral lipid emulsions for parenteral nutrition: random effects metaanalysis (Continued)

\begin{tabular}{llll}
\hline Non-ICU patients & 4 & 373 & $-4.97(-9.62,-0.32)^{*}$ \\
Serum bilirubin, overall MD & 4 & 520 & $0.03(-0.33,0.40)$ \\
ICU patients & 3 & 321 & $0.12(-0.42,0.65)$ \\
Non-ICU patients & 1 & 199 & $-0.02(-0.17,0.13)$
\end{tabular}

Values in parentheses represent $95 \% \mathrm{Cl}$. ${ }^{*}$ Significant effect $(P<0.05)$. ALT, alanine aminotransferase; AST, aspartate aminotransferase; CRP, C-reactive protein; DHA,(\%) docosahexaenoic acid content in serum/cellular membranes; EPA, (\%) eicosapentaenoic acid content in serum/cellular membranes; (H) LOS, (Hospital) length of stay; ICU, intensive care unit; LTB, leukotrienes B ; LTB ratio, LTB5: LTB4; MD, mean difference; RR, relative risk; SMD, standardised mean difference.

increase in leukotriene B5 (LTB5), the absolute decrease of LTB4, and the significantly ameliorated LTB5: LBT4 ratio (Figure 6).

\section{Routine laboratory parameters}

The analysis indicates a significant reduction in serum ALT and AST, with n-3 PUFA-enriched emulsions in comparison to standard lipid emulsions. However, no significant differences between the interventions were detected in coagulation times, platelet count, serum levels of triglycerides, C-reactive protein (CRP), or bilirubin.

\section{Fixed effect models}

In addition to the differences detected by the random effects models, fixed effect models also indicate a significant effect of n-3 PUFA-enrichment regimens on AST levels in ICU patients; bilirubin, CRP, and triglycerides in the overall and ICU populations; docosahexaenoic acid (DHA) in non-ICU patients, and LTB4 in the overall population (data not shown).

\section{Discussion}

A total of 23 studies ( $\mathrm{n}=1,502$ patients; $\mathrm{n}=762$ admitted to the ICU) were included in our meta-analysis. Pooled data indicate important and significant positive effects of n-3 PUFA-enriched parenteral regimens over a wide range of outcomes in the selected patient populations. Subgroup analysis shows that the magnitude of these effects for some of the outcomes varied between ICU patients and elective surgery patients. In some cases, although the effect is estimated as statistically significant for the whole considered population, this is not true for one or both subgroups, very probably as a consequence of the reduced patient number in the analysed population. Some differences in laboratory parameters (bilirubin, triglycerides, CRP, LTB4; data not shown) were not statistically significant with the random effects model, but were statistically significant when analysed with the less conservative, fixed effects model. For five pre-specified outcomes, the incidence of systemic inflammatory response syndrome (SIRS), bleeding events, international normalized ratio (INR), bleeding time, and creatinine clearance, data were qualitatively or quantitatively insufficient to obtain a metaanalytic effect estimate.

There are no clear data from RCTs on optimum doses, while a case series analysis indicates that the optimal dose may be related to the diagnosis [29]. Dosages used are mainly related to body weight, and if anything can be

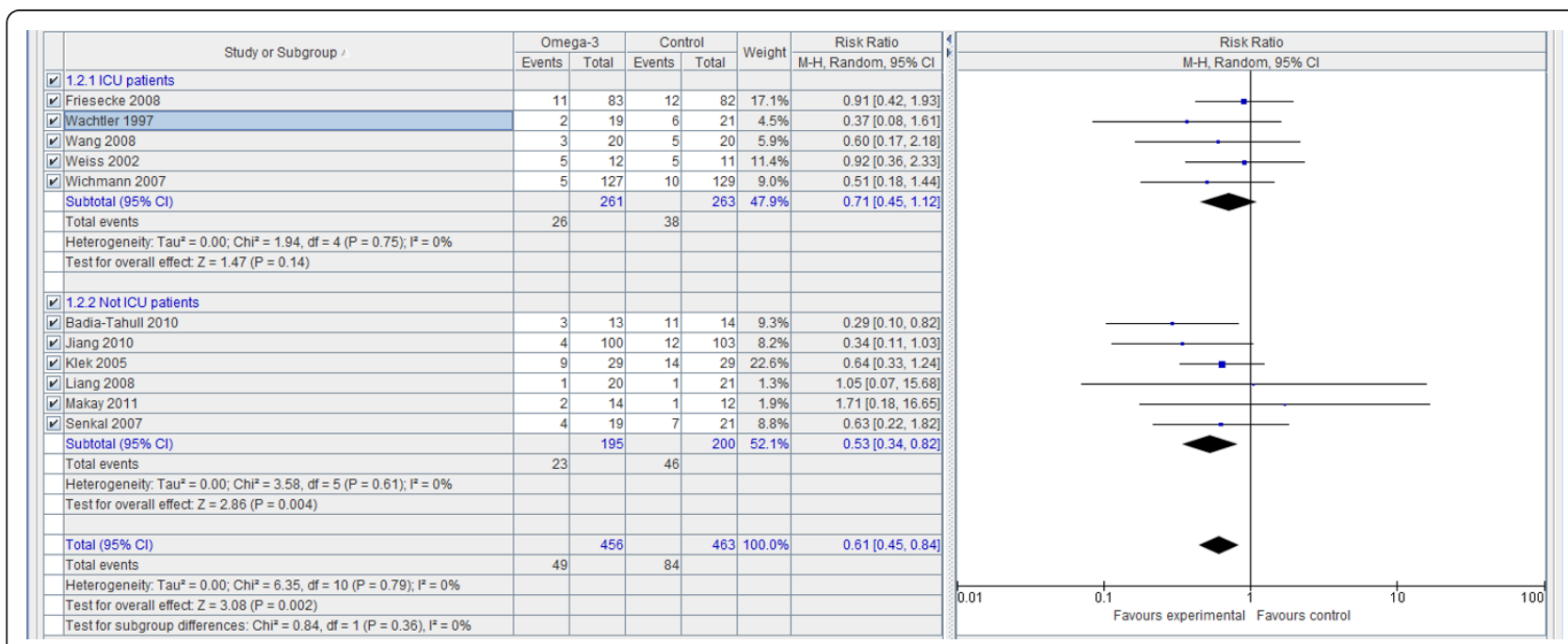

Figure 1 Infection rate: random effects meta-analysis and forest plot. Squares represent individual study mean of the effect measure, diamonds represent its pooled estimates. 


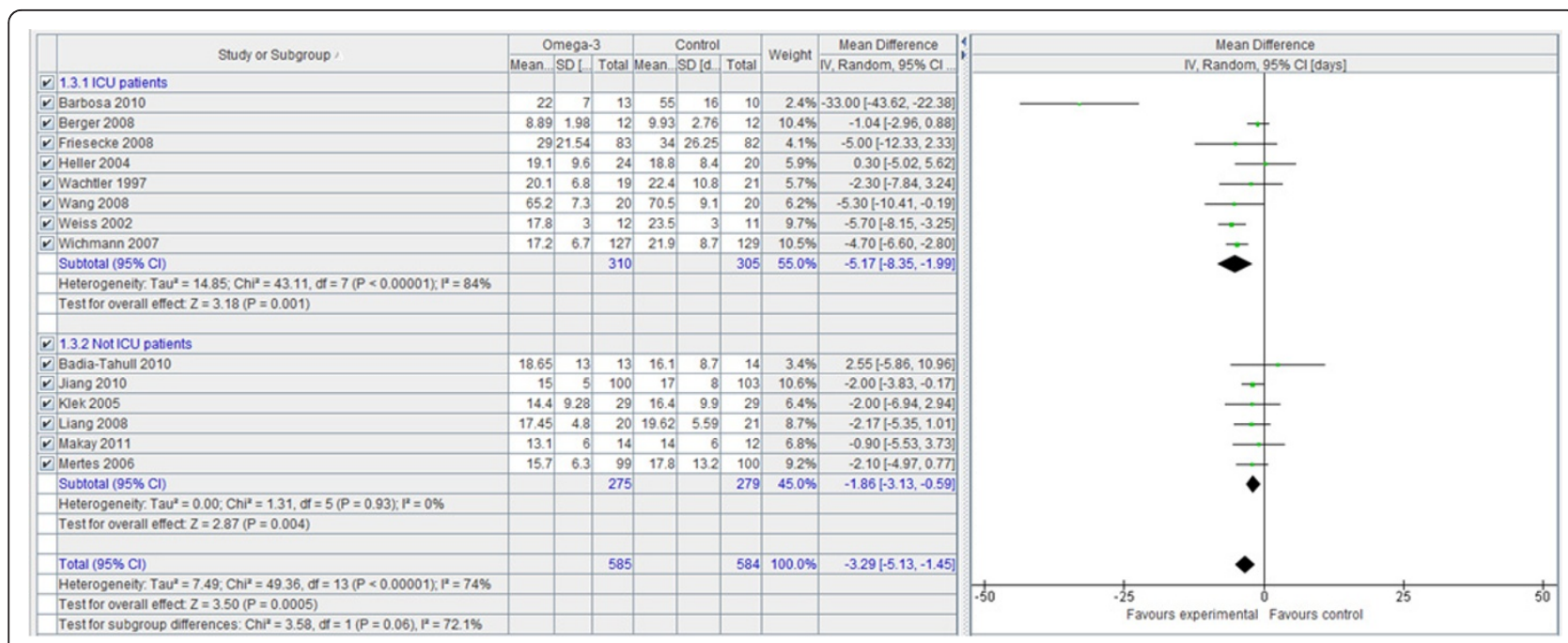

Figure 2 Hospital length of stay: random effects meta-analysis and forest plot. Squares represent individual study mean of the effect measure, diamonds represent its pooled estimates.

observed from existing data, the dose at least, must exceed 0.1 to $0.15 \mathrm{~g} / \mathrm{Kg} / \mathrm{d}$ fish oil. In order to address this question, a clinical trial for optimal dose determination has been designed and is being conducted (FOILED study: ClinicalTrials.gov NCT01146821). Regarding the optimal moment for starting parenteral lipids (pre- and/ or post-event), and possible effects on outcome, there are no inferential data, although logical thinking may suggest the earliest possible intervention.

The available pooled evidence in the present study on 3 PUFA-enriched parenteral regimens, in particular EPA and DHA, in surgical and ICU patients presenting with inflammatory conditions, indicates that their use is safe and effective in reducing the morbidity burden and the required hospitalization period. Moreover, results suggest that the hypothesised mechanism by which these effects are attained is plausible.
With n-3 PUFA-enrichment, the content in phospholipids clearly shifts its balance towards the $\mathrm{n}-3$ series, as indicated by relevant and statistically significant increases in EPA and DHA levels, without a significant decrease in AA, which is reassuring in light of the important physiological functions modulated by the latter. The more favourable balance of $n-3$ versus $n-6$ PUFAs is reflected in the increased release of less pro-inflammatory leukotrienes, such as LTB5, and particularly in the ratio between these and more pro-inflammatory cell messengers (LTB5/LTB4), and also by the reduction in cytokines, such as IL-6, and of the inflammation marker CRP (significantly reduced according to the fixed effects model, but with a non-significant trend towards reduced levels also in the more conservative random effects model). Actually, regarding the velocity of IL-6 reduction after peaking, there is more evidence in favor of $n-3$

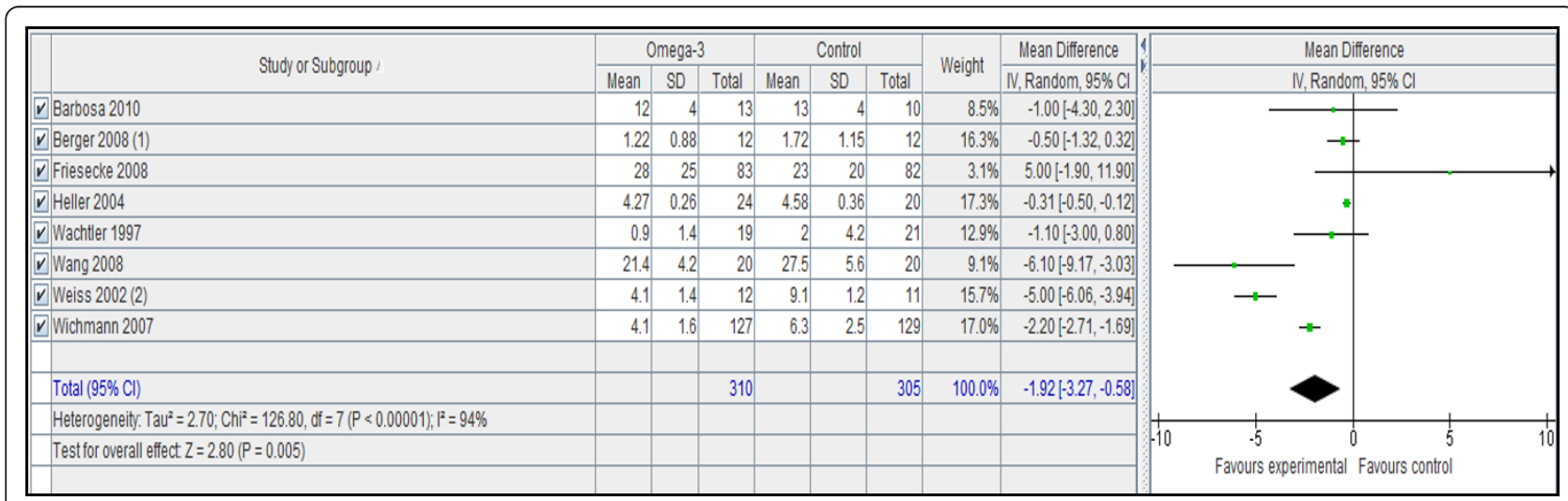

Figure 3 ICU length of stay: random effects meta-analysis and forest plot. Squares represent individual study mean of the effect measure, diamonds represent its pooled estimates. 


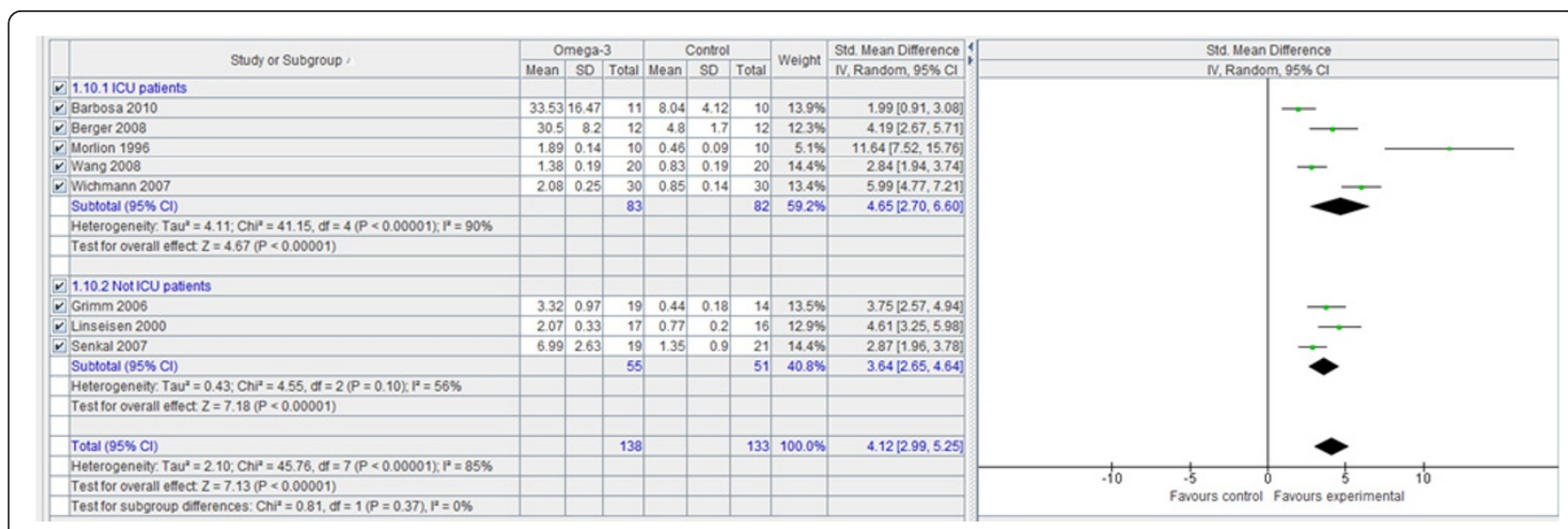

Figure 4 EPA concentration in plasma phospholipids: random effects meta-analysis and forest plot. EPA: eicosapentaenoic acid. Squares represent individual study mean of the effect measure, diamonds represent its pooled estimates.

PUFAs-enriched regimens than was possible to include in the quantitative data pooling: Jiang et al. [21], Wachtler et al. [15] and Weiss et al. [27] all report that IL-6 was increased in controls, and reduced in patients receiving n-3 PUFA-enriched regimens, and Wang et al. [16] report a significant difference in favour of n-3 PUFAenriched regimens, but none of these reports included adequate quantitative data.

Lung gas exchange assessed by the oxygenation index is better-preserved or improved in patients receiving $n$ 3 -enriched lipid emulsions than with standard lipid emulsions. As for safety of use, no significant differences among treatment groups could be detected in terms of coagulation parameters, either for laboratory markers, or the clinical outcome of blood transfusion requirements. The same holds true for renal function: serum creatinine and urea were not significantly different among treatment groups, and if anything can be deduced from the data, this is a trend towards less impairment of kidney function with n-3 PUFA-enriched emulsions. Liver enzymes are significantly less increased in patients receiving $\mathrm{n}-3$ PUFA-enriched emulsions than in those treated with standard lipid emulsions, suggesting a possible hepatoprotective action of fish oil components, which should be studied further.

The clinical results obtained are consistent with laboratory findings: although mortality is not significantly affected, there is a clear advantage in terms of infective complications and a relevant improvement in recovery times, as indicated by the significant reductions in the ICU and hospital LOS. There is a not a significant trend towards decreased mortality: possible explanations of the failure to show a significant effect include the low mortality risk in elective surgery patients $(<5 \%$ in the considered control groups) and the low overall patient numbers for high-mortality studies. However, the absence of a mortality difference increases the value of the LOS reduction, as it cannot be argued that this effect is a consequence of increased mortality.

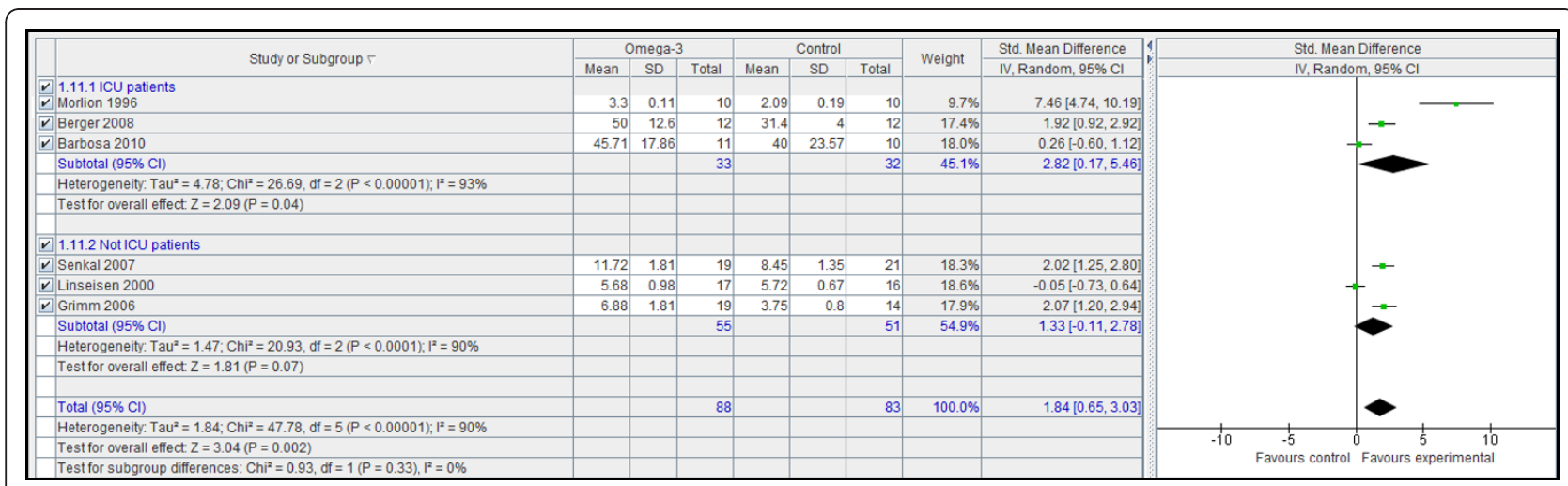

Figure 5 DHA concentration in plasma phospholipids: random effects meta-analysis and forest plot. DHA: docosahexaenoic acid. Squares represent individual study mean of the effect measure, diamonds represent its pooled estimates. 


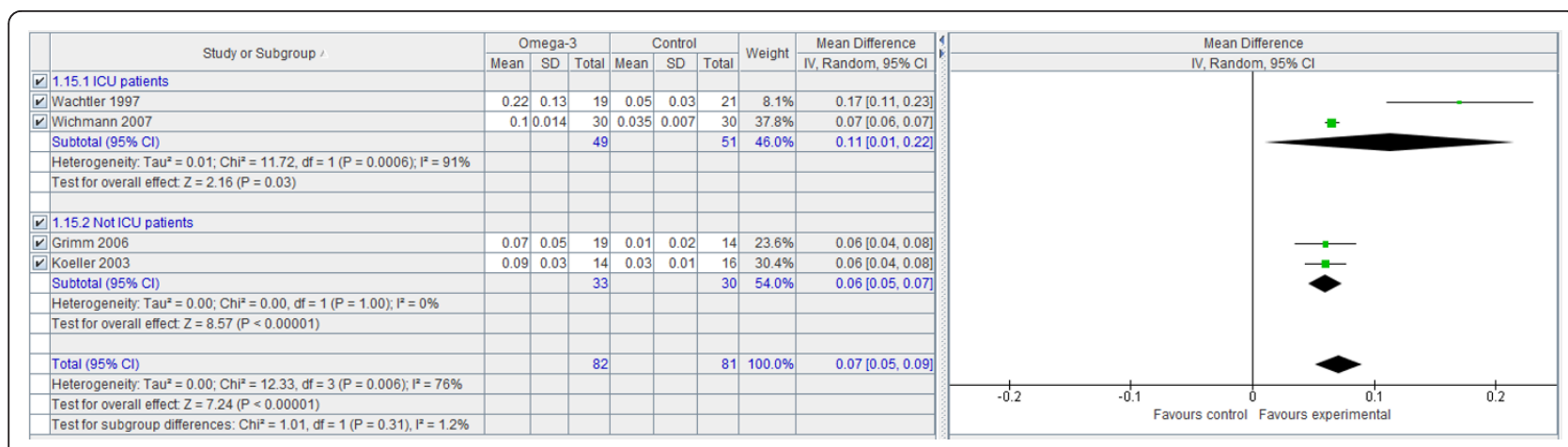

Figure 6 LTB5/LTB4 production: random effects meta-analysis and forest plot. LT: leukotriene. Squares represent individual study mean of the effect measure, diamonds represent its pooled estimates.

The fact that these results were obtained in studies in which different formulations of n-3 PUFAs were compared with a range of alternative lipid emulsions further strengthens the concept of using lipid emulsions that include the n-3 PUFA EPA and DHA. Our results confirm and extend the scope of those obtained in the earlier analysis in surgical patients by Chen et al. [5]. We included a greater number of studies and also evaluated data collected from patients admitted to ICU. The only relevant difference between outcomes considered in both analyses is the reduced leukocyte LTB4 production, which reached statistical significance with the fixed effects model.

\section{Conclusions}

In conclusion, these results confirm previous findings in surgical patients and extend them to the ICU population: the body of available evidence indicates that the use of n-3 PUFA-enriched parenteral nutrition is safe and effective in reducing the infection rate and hospital/ ICU stay in surgical patients, and that these benefits also apply to ICU patients. Other beneficial effects included reduced markers of inflammation, improved lung gas exchange, liver function, antioxidant status and fatty acid composition of plasma phospholipids, and a trend towards less impairment of kidney function.

\section{Key messages}

- Enriching conventional lipid emulsions with n-3 PUFA results in a statistically and clinically significant reduction in the infection rate and the length of stay in the ICU, and in postsurgical patients receiving parenteral nutrition.

- Mortality is not decreased, possibly because of the low mortality risk in the patient group as a whole.

- n-3 PUFA-enriched parenteral nutrition regimens are well-tolerated and there is a trend towards less impairment of kidney function, as well as significantly improved liver function and lung gas exchange.

\section{Additional material}

Additional file 1: Figure S1. Prisma flowchart of study selection.

\section{Abbreviations}

AA: arachidonic acid; ALT: alanine aminotransferase; AST: aspartate aminotransferase; CRP: C-reactive protein; DHA: docosahexaenoic acid; EPA: eicosapentaenoic acid; ICU; intensive care unit; LCT: long-chain triglyceride; LTB: leukotriene B; LOS: length of stay; MCT: medium-chain triglyceride; Ol: oxygenation index; PT: prothrombin time; PPT: partial thromboplastin time; PUFA: polyunsaturated fatty acids; RCT: randomized clinical trial; RR: relative risk; SMD: standardised mean difference.

\section{Acknowledgements}

Orietta Zaniolo, PharmD, helped in data extraction and the consistency check. Julia Balfour, Medical Writer, Dundee, Scotland provided assistance with the preparation of the manuscript.

\section{Author details}

'AdRes HE\&OR, Piazza Carlo Emanuele II 19, I-10123, Turin, Italy. ${ }^{2}$ Lung Transplant Program, Internal Medicine, Pulmonary Medicine, Intensive Care Medicine, Sleep Medicine. Department of Internal Medicine, Justus-Liebig University Giessen, Klinikstrasse 36, D-35392, Giessen, Germany. ${ }^{3}$ Internal Medicine, Università La Sapienza, Via del Policlinico, 155, I-00161, Rome, Italy. ${ }^{4}$ Clinic for Anaesthesiology and Intensive Therapy, University Dresden, Fetscherstraße 74, D-01307 Dresden, Germany.

\section{Authors' contributions}

LP conceived the study, extracted clinical data, performed the statistical analysis and drafted the manuscript. KM participated in the design of the study, reviewed the literature and helped to draft the manuscript. MM participated in the design of the study, reviewed the literature and helped to draft the manuscript. AH participated in the design of the study, reviewed the literature and helped to draft the manuscript. All authors read and approved the final manuscript.

\section{Competing interests}

Dr. Lorenzo Pradelli is co-owner and employee of AdRes, which has received project funding by Fresenius Kabi. Prof. Axel Heller received speaker honoraria and project funding by BBraun, Melsungen, Germany and by Fresenius- Kabi, Bad Homburg, Germany. Prof. Maurizio Muscaritoli received speaker honoraria by Baxter, BBraun, and Fresenius Kabi. K. Mayer received fees for product neutral lectures and compensation for travel costs from Abbott, Baxter, BBraun, Fresenius Kabi, Nestle, Pfizer.

Received: 19 June 2012 Revised: 19 June 2012 Accepted: 4 October 2012 Published: 4 October 2012 


\section{References}

1. Dyerberg J, Bang HO, Hjorne N: Fatty acid composition of the plasma lipids in Greenland Eskimos. Am J ClinNutr 1975, 28:958-966.

2. Dyerberg J, Bang HO, Stoffersen E, Moncada S, Vane JR: Eicosapentaenoic acid and prevention of thrombosis and atherosclerosis? Lancet 1978, 2:117-119.

3. Grimm H, Tibell A, Norrlind B, Blecher C, Wilker S, Schwemmle K: Immunoregulation by parenteral lipids: impact of the $n-3$ to $n-6$ fatty acid ratio. JPEN 1994, 18:417-421.

4. Fürst $P$, Kuhn KS: Fish oil emulsions: what benefits can they bring? Clin Nutr 2000, 19:7-14

5. Chen B, Zhou Y, Yang P, Wan HW, Wu XT: Safety and efficacy of fish oilenriched parenteral nutrition regimen on postoperative patients undergoing major abdominal surgery: a meta-analysis of randomized controlled trials. JPEN 2010, 34:387-394.

6. Antébi H, Mansoor O, Ferrier C, Tétégan M, Morvan C, Rangaraj J, Alcindor LG: Liver function and plasma antioxidant status in intensive care unit patients requiring total parenteral nutrition: comparison of 2 fat emulsions. JPEN 2004, 28:142-148.

7. Barbosa VM, Miles EA, Calhau C, Lafuente E, Calder PC: Effects of a fish oil containing lipid emulsion on plasma phospholipid fatty acids, inflammatory markers, and clinical outcomes in septic patients: a randomized, controlled clinical trial. Crit Care 2010, 14:R5.

8. Berger MM, Tappy L, Revelly JP, Koletzko BV, Gepert J, Corpataux JM, Cayeux MC, Chiolero RL: Fish oil after abdominal aorta aneurysm surgery. Eur J Clin Nutr 2008, 62:1116-1122.

9. Friesecke S, Lotze C, Köhler J, Heinrich A, Felix SB, Abel P: Fish oil supplementation in the parenteral nutrition of critically ill medical patients: a randomised controlled trial. Intensive Care Med 2008, 34:1411-1420.

10. Heller AR, Rössel T, Gottschlich B, Tiebel O, Menschikowski M, Litz RJ, Zimmermann T, Koch T: Omega-3 fatty acids improve liver and pancreas function in postoperative cancer patients. Int J Cancer 2004, 111:611-616.

11. Morlion BJ, Torwesten E, Lessire H, Sturm G, Peskar BM, Fürst P, Puchstein C. The effect of parenteral fish oil on leukocyte membrane fatty acid composition and leukotriene-synthesizing capacity in patients with postoperative trauma. Metabolism 1996, 45:1208-1213.

12. Piper SN, Schade I, Beschmann RB, Maleck WH, Boldt J, Röhm KD: Hepatocellular integrity after parenteral nutrition: comparison of a fishoil-containing lipid emulsion with an olive-soybean oil-based lipid emulsion. Eur J Anaesthesiol 2009, 26:1076-1082.

13. Roulet $M$, Frascarolo P, Pilet $M$, Chapuis G: Effects of intravenously infused fish oil on platelet fatty acid phospholipid composition and on platelet function in postoperative trauma. JPEN 1997, 21:296-301.

14. Sabater J, Masclans JR, Sacanell J, Chacon P, Sabin P, Planas M: Effects of an omega-3 fatty acid-enriched lipid emulsion on eicosanoid synthesis in acute respiratory distress syndrome (ARDS): A prospective, randomized, double-blind, parallel group study. Nutr Metab (Lond) 2011 8:22.

15. Wachtler $P$, König W, Senkal M, Kemen M, Köller M: Influence of a total parenteral nutrition enriched with omega- 3 fatty acids on leukotriene synthesis of peripheral leukocytes and systemic cytokine levels in patients with major surgery. J Trauma 1997, 42:191-198.

16. Wang X, Li W, Zhang F, Pan L, Li N, Li J: Fish oil-supplemented parenteral nutrition in severe acute pancreatitis patients and effects on immune function and infectious risk: a randomized controlled trial. Inflammation 2009, 32:304-309.

17. Weiss G, Meyer F, Matthies B, Pross M, Koenig W, Lippert H: Immunomodulation by perioperative administration of $\mathrm{n}-3$ fatty acids. $B$ J Nutr 2002, 87:589-94.

18. Wichmann MW, Thul P, Czarnetzki HD, Morlion BJ, Kemen M, Jauch KW: Evaluation of clinical safety and beneficial effects of a fish oil containing lipid emulsion (Lipoplus, MLF541): data from a prospective, randomized, multicenter trial. Crit Care Med 2007, 35:700-706

19. Badía-Tahull MB, Llop-Talaverón JM, Leiva-Badosa E, Biondo S, FarranTeixidó L, Ramón-Torrell JM, Jódar-Masanes R: A randomised study on the clinical progress of high-risk elective major gastrointestinal surgery patients treated with olive oil-based parenteral nutrition with or without a fish oil supplement. Br J Nutr 2010, 104:737-741.
20. Grimm H, Mertes N, Goeters C, Schlotzer E, Mayer K, Grimminger F, Fürst P: Improved fatty acid and leukotriene pattern with a novel lipid emulsion in surgical patients. Eur J Nutr 2005, 45:55-60.

21. Jiang ZM, Wilmore DW, Wang XR, Wei JM, Zhang ZT, Gu ZY, Wang S, Han SM, Jiang H, Yu K: Randomized clinical trial of intravenous soybean oil alone versus soybean oil plus fish oil emulsion after gastrointestinal cancer surgery. Br J Surg 2010, 97:804-809.

22. Kłek S, Kulig J, Szczepanik AM, Jedrys J, Kołodziejczyk P: The clinical value of parenteral immunonutrition in surgical patients. Acta Chir Belg 2005, 105:175-179

23. Koeller M, Senkal M, Kemen M, Koenig W, Zumtobel V, Muhr G: Impact of omega-3 fatty acid enriched TPN on leukotriene synthesis by leukocytes after major surgery. Clinical Nutrition 2003, 22:59-64.

24. Bin Liang, Shan Wang, Ying-Jiang Ye, Xiao-Dong Yang, You-Li Wang, Jun Qu, Qi-Wei Xie, Mu-Jun Yin: Impact of postoperative omega-3 fatty acid-supplementedparenteral nutrition on clinical outcomes andimmunomodulations in colorectal cancer patients. World J Gastroenterol 2008, 14:2434-2439.

25. Linseisen J, Hoffmann J, Lienhard S, Jauch KW, Wolfram G: Antioxidant status of surgical patients receiving TPN with an omega-3-fatty acidcontaining lipid emulsion supplemented with alpha-tocopherol. Clin Nutr 2000, 19:177-184.

26. Makay O, Kaya T, Firat O, Sozbilen M, Caliskan C, Gezer G, Uyar M, Ersin S: $\omega-3$ Fatty acids have no impact on serum lactate levels after major gastric cancer surgery. JPEN 2011, 35:488-492.

27. Mertes N, Grimm H, Fürst P, Stehle P: Safety and efficacy of a new parenteral lipid emulsion (SMOFlipid) in surgical patients: a randomized, double-blind, multicenter study. Ann Nutr Metab 2006, 50:253-259.

28. Senkal M, Geier B, Hannemann M, Deska T, Linseisen J, Wolfram G, Adolph M: Supplementation of omega-3 fatty acids in parenteral nutrition beneficially alters phospholipid fatty acid pattern. JPEN 2007, 31:12-17.

29. Heller AR, Rössler S, Litz RJ, Stehr SN, Heller SC, Koch R, Koch T: Omega-3 fatty acids improve the diagnosis-related clinical outcome. Crit Care Med 2006, 34:972-979.

doi:10.1186/cc11668

Cite this article as: Pradelli et al:: $\mathrm{n}-3$ fatty acid-enriched parenteral nutrition regimens in elective surgical and ICU patients: a meta-analysis. Critical Care 2012 16:R184.

\section{Submit your next manuscript to BioMed Central and take full advantage of:}

- Convenient online submission

- Thorough peer review

- No space constraints or color figure charges

- Immediate publication on acceptance

- Inclusion in PubMed, CAS, Scopus and Google Scholar

- Research which is freely available for redistribution

Submit your manuscript at www.biomedcentral.com/submit
C Biomed Central 